\title{
Short Communication \\ Measuring the torque required to cause vertebral dislocation in cattle tails
}

\author{
R Laven* and MC Jermy ${ }^{\dagger \S}$ \\ ${ }^{*}$ School of Veterinary Science, Massey University, Tennent Drive, Palmerston North 4474, New \\ Zealand \\ $\dagger$ Department of Mechanical Engineering, University of Canterbury, Private Bag 4800, Christchurch \\ 8140, New Zealand \\ § Author for Correspondence. Email: mark.jermy@canterbury.ac.nz
}

\begin{abstract}
AIM: To estimate, ex vivo, the torque required to cause vertebral dislocation of cattle tails.

METHODS: Five tails from dairy cows were sourced from a slaughterhouse, severed at the junction between the sacrum and the first caudal vertebra. Within 2 hours of slaughter the severed end of each tail was clamped in a vice and a steel collar was placed halfway along the tail. A torque wrench was attached to the steel collar, which was then rotated to produce an audible and palpable vertebral dislocation, and the torque at the time of the break was recorded .

RESULTS: The maximum torque required to break a tail was $20 \mathrm{Nm}$, the minimum was $9.8 \mathrm{Nm}$, and the uncertainty was $4.9 \mathrm{Nm}$.

CONCLUSIONS: The torque required to break a cow's tail is unlikely to be applied accidentally if cattle are handled following recommended best practice. Thus if cattle on a farm can be shown to have broken tails due to manipulation by farm staff, cattle handling is not meeting the recommended best practice of the New Zealand Dairy Cattle Code of Welfare.

KEY WORDS: Cattle; tail break; torque; abusive injury; control
\end{abstract}

\section{Introduction}

Tail injuries can cause significant pain and distress to dairy cattle. Broken tails, where damage to the tail results in tearing of the caudal fascia, swelling around the intervertebral joints or deviation of the tail, due to dislocation of those joints, are associated with welfare compromise at the time of 
the injury and subsequently because the damage and restriction of movement can result in the loss of the functionality of the tail (Hemsworth et al. 1995; de Oliveira and Keeling 2018).

There are very few published reports of the prevalence of broken tails in dairy cattle. The only peerreviewed survey is that reported by Zurbrigg et al. (2005). Between March and September 2003 they measured the prevalence of broken tails on 317 dairy farms in Ontario, Canada, that used tiestalls. They reported that, at the farm level, prevalence of broken tails ranged from 0 to $50 \%$, with 196/317 (62\%) farms having no broken tails and only 17 (5\%) having a prevalence $>15 \%$. Based on these results, the authors stated that farms should be able to achieve $<1 \%$ of broken tails. This conclusion is consistent with the results of a survey of 23 pasture-based dairy farms in Uruguay, of which $16(70 \%)$ had $<1 \%$ broken tails, four had a prevalence between 1 and $5 \%$, and three $>5 \%$ (de Wolf 2010).

Nevertheless broken tails are a clear, easily detected indicator of poor welfare (Grandin 2017), so measurement of the proportion of broken tails can form part of a welfare assessment programme for dairy cattle. However such assessments do not routinely include the proportion of broken tails; of five assessment protocols evaluated by Winckler (2006), broken tails were included in only one, and broken tails are not included in the Welfare Quality assessment protocol for cattle (Anonymous 2009), which is probably the gold standard for welfare assessments (Laven and Fabian 2016). Moreover, only one of 21 respondents who were all Europe-based experts in animal welfare assessment, reported that they included broken tails as an animal-based welfare measure (Neilsen et al. 2014).

None of the welfare assessment programmes that do include broken tails have published data on prevalence. Nevertheless the targets set for the percentage of broken tails are close to the $1 \%$ recommended by Zurbrigg (2005). For example, in the United Kingdom, the RSPCA welfare standards state that it is possible to maintain a herd with no broken tails (Anonymous 2018c) and the American Humane standards state that $\geq 98 \%$ of cattle must have undamaged, unbroken tails (Anonymous 2017). Furthermore, assessment schemes which do not set explicit targets, such as that provided by the University of California, Davis, suggest that the prevalence of broken tails is rare (Anonymous 2018a).

The situation appears to be different in New Zealand. Anecdotal reports suggest that tail breaking seems to be an increasing problem on dairy farms in New Zealand. A preliminary report of 4 years of data, from dairy farms participating in a voluntary herd welfare and productivity monitoring programme, stated that in the 2014/2015 season the lactation incidence of new cases of broken tails was $0.06 \%$ whereas in $2017 / 18$ the lactation incidence was $4.5 \%$ (Bryan et al. 2019). In addition, 
over the 4 years $\sim 10 \%$ of the cows examined had broken tails, a figure much higher than the $1 \%$ target recommended by Zurbrigg (2005), although the percentage of farms reported as having no broken tails was $70-82 \%$ (depending on the year), which was higher than the $62 \%$ reported by Zurbrigg et al. (2005). The percentage of cows with broken tails reported by Bryan et al. (2019) is also higher than the median herd prevalence of $4.8 \%$ (first quartile $2.0 \%$, third quartile $9.2 \%$ ) in the United Kingdom, for cows with tail injuries on 51 farms, in an unpublished report (S. Collins, ${ }^{1}$ pers. comm.).

There are no published investigations of the causes of broken tails. It has been suggested that they may be caused by mechanical damage, inappropriate handling or other causes (Anonymous, 2018b). For cows kept in tie stalls, Zurbrigg et al. (2005) stated that broken tails may result from the tail being stepped on or through forceful manipulation of the tail by dairy producers, and they linked the negative association between tie rail height and prevalence of broken tails to impatient producers being more likely to twist the tail if the tie rail height was low. In pasture-based cattle, de Wolf (2010) concluded that staff impatience was the cause of a high prevalence of broken tails.

The link between staff actions and broken tails is made because, in cattle, manipulation of the tail is commonly used to control an animal. The main structure of the bovine tail is the caudal vertebrae, joined by fibrocartilaginous intervertebral discs, and the muscles of the tail, which are enveloped by a strong caudal fascia. The superficial layer of this caudal fascia is thin and attached to the skin, the deep layer is thicker and attached to the caudal vertebrae (Nickel et al. 1992). If the tail of the cow is lifted or gently twisted in a U-shape the cow will move forward. This process should require very little force or strength. Furthermore, to prevent a cow from kicking, the tail can be lifted straight up (Figure 1). This allows for safer examination of the udder and surrounding area, as well as administration of treatments. Again this should not require significant force, as the response is not dependent on causing the animal pain. Nevertheless, because of the potential for poorly applied manipulation to cause pain and distress, recommended best practice in the New Zealand Dairy Cattle Code of Welfare is that tails are neither twisted nor lifted (Anonymous 2018d).

Breaking tails is one of the commonest issues in cattle-related welfare complaints that come to court in New Zealand (P. Timmer-Arends, ${ }^{2}$ pers. comm.) with some farms having $50 \%$ of cattle with broken tails (R. Laven, unpublished data). In most of such cases the point of breakage is in the middle third of the tail and there is usually eyewitness evidence of tails being broken or at least repeatedly twisted by farm staff. A common defence is that the actions seen were normal practice and that tails are very easily broken. As with most areas relating to broken tails, there is no

${ }^{1}$ S. Collins, RSPCA Assured, Horsham, UK

${ }^{2}$ P. Timmer-Arends, MPI, Wellington, New Zealand 
published quantitative information about the force required to cause the injuries seen in cows with broken tails. The aim of this study was to estimate, ex vivo, the torque required to cause such injury.

\section{Materials and methods}

Five tails from dairy cows were sourced from a slaughterhouse in Christchurch (Canterbury, NZ)., They were severed at the junction between the sacrum and the first caudal vertebra and were used within 2 hours of slaughter. There were no signs of rigor mortis and the temperature of the tails was $21^{\circ} \mathrm{C}$ at the commencement of the tests.

The test was designed to measure the torque required to dislocate a vertebral joint by a person grasping the tail with one hand and pushing the free end of the tail towards the animal's back. Torque is the rotational effort which is equivalent to the force applied at the point of application multiplied by the length of the lever (the distance between the point of application of force and the pivot). For example, the torque applied when lifting $2 \mathrm{~kg}$ at the end of a $70 \mathrm{~cm}$ lever is $14 \mathrm{Nm}$.

The severed end of each tail was wrapped in a plastic bag secured with a rubber band and then clamped in a vice between two pieces of medium-density fibreboard, which were $120 \mathrm{~mm}$ in length. The tail was rotated through $90^{\circ}$ so that bending the tail in the horizontal plane during the test simulated pushing it in a vertical plane in a standing animal (Figure 2).

The distance was measured between the vice (the edge of the fibreboard distal to the severed end of the tail) and the most distal vertebra (the last bony or stiff section of the tail that could be felt). A steel collar was then placed on the tail halfway along this measured distance. The collar was fabricated from a $64 \mathrm{~mm}$ length of $40 \times 40 \mathrm{~mm}$ square hollow section steel that had a wall thickness of $4 \mathrm{~mm}$, with a bolt welded to the outside (Figure 2).

The torque required to break the tail was measured using a beam-type torque wrench (RAM-PRO, Taiwan) via a socket that fitted over the bolt head on the steel collar. The tester (MJ) held the collar in place with his left hand, and rotated the torque wrench with his right hand (Figure 2) until a sound was heard that indicated the tail had broken, and the resistance was felt to slacken, which occurred simultaneously. The scale on the torque wrench was recorded using a video camera (Sony HDV Handycam (Sony, Tokyo, Japan)) during this process. The maximum torque observed, and the torque at the time of the break, were both recorded. The zero-load offset of $5.0 \mathrm{Nm}$ was subtracted from the torque reading, to give the torque value required to produce the break. The mass of each tail was measured on an electronic scale (Mettler-Toledo, Columbus, OH, USA) after it was tested. 
Uncertainties for the measurements were also determined. For the mass of the tail, the uncertainty was the resolution of the electronic scales. For the distances, the uncertainties arose because the tail was not always straight, and were estimated from the difference between measurements along the surface of the tail and a straight line between the ends when taut. For torque readings, the uncertainty arose because of parallax as it was not possible to ensure that the camera was always directed at $90^{\circ}$ to the scale, and one tail (Tail 2) was twisted about its axis slightly before the torque wrench was applied.

\section{Results}

The results for each tail are presented in Table 1. The maximum torque required to break a tail was $20 \mathrm{Nm}$, and the minimum $9.8 \mathrm{Nm}$, with an uncertainty of $4.9 \mathrm{Nm}$.

\section{Discussion}

This study has, for the first time, provided information about the force required to dislocate a vertebral joint in a cow's tail. The torque required varied between tails, probably due to variations in anatomy from one individual to another, and in position of the metal collar with respect to the intervertebral discs.

Torque is equivalent to the force applied at the point of application multiplied by the lever arm length. For a broken tail, the length of the lever is the distance from the point at which the tail is grasped and pushed to the point of breakage, and the breakage occurs close to the effective pivot point. In most cases observed by the authors, the point of breakage is in the middle third of the tail, i.e. approximately $20-40 \mathrm{~cm}$ from the free end of a tail approximately $60 \mathrm{~cm}$ long. If the tail is grasped in the manner shown in Figure 1, and the break occurs in this middle third, the lever arm length will be not more than $30 \mathrm{~cm}$. To achieve the minimum breaking torque measured in this study $(9.8 \mathrm{Nm})$, application of a force of $33 \mathrm{~N}(9.8 \mathrm{Nm} / 0.3 \mathrm{~m})$ would be required. If the lever arm length is shorter, a greater force is needed to achieve the same torque. In humans, mean maximal force when lifting directly upwards at shoulder level, at maximum reach distance, is $81 \mathrm{~N}$, increasing to $180 \mathrm{~N}$ when pushing horizontally (Haselgrave et al. 1997). Thus the force required to break a cows tail in this manner is $\sim 18-40 \%$ of the average maximum force that can be applied under those circumstances. It is thus unlikely that if a cow's tail is lifted in this way, in a controlled and careful manner, that breakage can occur unintentionally.

Thus although these data are limited, being based on only five tails, they show clearly that significant force is required to result in dislocation of tail vertebrae. The experiment also demonstrated that the dislocation resulting from applying an excess force to a tail did make a 
detectable noise and change in resistance. Clearly, in a milking shed the noise might be more difficult to hear because of the increased background noise, but in other circumstances away from the milking shed it is more likely that the sound will be heard, and could be reported to a veterinarian. Currently, very few broken tails are promptly reported to veterinarians, although some are detected at a later date when treatment is unlikely to result in improvement in cow welfare.

Significant questions remain about broken tails in New Zealand. The high number of anecdotal reports, and a prevalence on many farms above the $1 \%$ recommended overseas, shows that a properly planned study of the prevalence of broken tails is needed, using a validated protocol. In addition, such a study should examine risk factors for tail breaking to facilitate determination and mitigation of the range of probable causes of this source of cattle welfare compromise. This will be useful both for improving cow welfare and will make it clearer when forceful manipulation is the main issue causing broken tails.

Important limitations of this study are that force was applied only at a single point and direction, but other grasps are possible; the number of tails tested was small and the tests were performed postmortem and at room temperature. Tissue properties may change post-mortem, but the short time between slaughter and test limited any deterioration.

In conclusion, this study determined, using an ex vivo model, that the maximum torque required to break a cow's tail was $20 \mathrm{Nm}$ and the minimum $9.8 \mathrm{Nm}$. This torque is unlikely to be applied accidentally if cattle are handled following recommended best practice. Thus if cattle on a farm can be shown to have broken tails due to manipulation by farm staff, cattle handling is not meeting the recommended best practice of the New Zealand Dairy Cattle Code of Welfare.

\section{Acknowledgements}

We would like to thank Mr Richard Notley of the New Zealand Ministry for Primary Industries for commissioning this work and sourcing the tails.

\section{References}

*Anonymous. Welfare Quality Assessment Protocol for Cattle. http://www.welfarequalitynetwork.net/media/1088/cattle_protocol_without_veal_calves.pdf (accessed 14 September 2018). Welfare Quality Network, Uppsala, Sweden, 2009

* Anonymous. Animal Welfare Standards for Dairy Cattle.

http://www.humaneheartland.org/index.php?option=com_content\&view=article\&id=3\&Itemid $=106 \& \mathrm{j}$ smallfib $=1 \& \mathrm{dir}=\mathrm{JSROOT} /$ Animal + Welfare + Full + Standards $+\% 2 \mathrm{~B}+$ Supplements $\&$ dow 
nload_file=JSROOT/Animal+Welfare+Full + Standards $+\% 2 B+$ Supplements/Dairy + Cattle + Full +Standards.pdf (accessed 14 September 2018). American Humane, Washington, DC, USA, 2017

*Anonymous. Broken Tail. https://www.ucdcowcalfassessment.com/broken-tail.html (accessed 14 September 2018). University of California Davis, Davis, CA, USA, 2018a

*Anonymous. Dairy Cows: Broken Tails. http://www.assurewel.org/dairycows/brokentails.html (accessed 9 October 2019). AssureWel, UK, 2018b

*Anonymous. RSPCA Welfare Standards for Dairy Cattle. https://science.rspca.org.uk/sciencegroup/farmanimals/standards/dairycattle (accessed 14 September 2018). RSPCA, UK, 2018c

*Anonymous. Code of Welfare: Dairy Cattle https://www.agriculture.govt.nz/dmsdocument/1415dairy-cattle-animal-welfare-code-of-welfare (accessed 9 October 2019). Ministry for Primary Industries, Wellington, NZ, 2018d

* Bryan M, Fruean SN, Moono P Tail damage and tail scoring in NZ dairy cows - what is normal? HoofPrint NZ: Official Newsletter of the Dairy Cattle Veterinarians Branch of the NZVA 36 No. 4, 10

de Oliveira D and Keeling LJ. Routine activities and emotion in the life of dairy cows: Integrating body language into an affective state framework. PLoS ONE 13(5): e0195674. 2018.

*de Wolf A. A welfare assessment system for dairy cows on pasture and the comparison to a welfare scoring system for cows in cubicles. https://dspace.library.uu.nl/handle/1874/44627 2010 (accessed 9 October 2019). PhD thesis, Utrecht University, Utrecht, Netherlands, 2010.

Grandin T. On-farm conditions that compromise animal welfare that can be monitored at the slaughter plant, Meat Science, Volume 132, 52-58, 2017

Haslegrave CM, Tracy MF and Corlett EN. Force exertion in awkward working posturesstrength capability while twisting or working overhead, Ergonomics, 40:12, 1335-1356, 1997

Hemsworth P H, Barnett J L, Beveridge L, and Matthews L R. The welfare of extensively managed dairy cattle: A review. Applied Animal Behaviour Science, 42(3), 161-182, 1995. 
Laven RA and Fabian J. Applying animal-based welfare assessments on New Zealand dairy farms: feasibility and a comparison with United Kingdom data New Zealand Veterinary Journal, Volume 64, Issue 4, pp 212-217, 2016

*Nielsen BH, Angelucci A, Scalvenzi A, Forkman B, Fusi F, Tuyttens FAM, Houe H, Blokhuis HJ, Sørensen JT, Rothmann J, et al. Use of animal based measures for the assessment of dairy cow welfare (ANIBAM) External Scientific Report EN-659. https://efsa.onlinelibrary.wiley.com/doi/10.2903/sp.efsa.2014.EN-659 (accessed 9 October 2019). EFSA, Parma, Italy, 2014

*Nickel R, Schummer A, Seiferle E, Tiefe Hals-, Rumpf-, Schwanzfaszie. Lehrbuch der Anatomie der Haustiere, Band 1 Bewegungsapparat. [Textbook of the Anatomy of Domestc Animals, Volume I: Musculoskeletal System] $6^{\text {th }}$ Edtn. Pp 336-7. Verlag Paul Parey, Berlin, Germany, 1992

*Winckler C. On-farm welfare assessment in cattle from basic concepts to feasible assessment systems. Proceedings of the 24th World Buiatrics Congress. Pp 493-501, 2006

Zurbrigg K, Kelton D, Anderson N, and Millman S. Stall dimensions and the prevalence of lameness, injury, and cleanliness on 317 tie-stall dairy farms in Ontario. The Canadian Veterinary Journal, 46(10), 902-909, 2005

* Non-peer-reviewed

Submitted 12 November 2018

Accepted for publication 8 October 2019

First published online [insert date] 
Table 1. Mass $(\mathrm{kg})$ and length $(\mathrm{cm})$ of tail (vice to tail tip), with location of torque wrench (cm relative to the vice holding the tail), and the torque required $(\mathrm{Nm})$ to produce a break, in five cows' tails collected after slaughter.

\begin{tabular}{lcccc}
\hline Tail number & Mass & Total length & Location of wrench & Torque required \\
\hline 1 & 1.255 & 76 & 38 & 15 \\
2 & 1.724 & 50 & 24 & 20 \\
3 & 1.251 & 60 & 30 & 9.8 \\
4 & 1.140 & 60 & 30 & 9.8 \\
5 & 1.813 & 60 & 30 & 15 \\
Uncertainty & \pm 0.005 & \pm 3 & \pm 1 & \pm 4.9 \\
\hline
\end{tabular}

ancertainties for measurements 
Figure 1. Photograph showing the method for restraining a cow by grasping the tail with one hand and pushing the free end of the tail towards the animal's back.

Figure 2. Photograph showing the position of a cow's tail for measurement of the torque required, ex vivo, to cause a break. The severed (sacral) end of the tail was held within a vice and a steel collar with attached torque wrench was placed half way between the vice and the most distal caudal vertebra. Note that, relative to the position on a standing animal, the tail has been rotated through $90^{\circ}$. 